American Journal of Pediatrics
2020; 6(4): $488-494$
http://www.sciencepublishinggroup.com/j/ajp
doi: 10.11648 /j.ajp.20200604.27
ISSN: $2472-0887$ (Print); ISSN: $2472-0909$ (Online)

\title{
Influence of Exercise on Body Composition and Patient Outcomes in a Multidisciplinary Pediatric Obesity Clinic
}

\author{
Rebecca Espinosa ${ }^{1}$, Kelly Hearne ${ }^{2}$, Ayoola Adigun ${ }^{2, *}$, Lisa Farkas ${ }^{3}$, Ryan Cranshaw $^{4}$, \\ Cara Boyarin ${ }^{4}$, Morgan Sawyer ${ }^{4}$, Debora Duro ${ }^{5}$ \\ ${ }^{1}$ Department of Graduate Medical Education, Salah Foundation Children's Hospital, Fort Lauderdale, USA \\ ${ }^{2}$ Department of Grants Administration, Broward Health Medical Center, Fort Lauderdale, USA \\ ${ }^{3}$ Broward Health Wellness Center, Broward Health Medical Center, Fort Lauderdale, USA \\ ${ }^{4}$ Department of Wellness and Cardiopulmonary Rehabilitation, Broward Health Medical Center, Fort Lauderdale, USA \\ ${ }^{5}$ Department of Pediatric Gastroenterology, Hepatology, and Nutrition, Florida Intestinal Rehabilitation Support and Treatment (FIRST) at \\ Salah Foundation Children Hospital, Broward Health Medical Center, Fort Lauderdale, USA
}

Email address:

aadigun@browardhealth.org (A. Adigun)

*Corresponding author

\section{To cite this article:}

Rebecca Espinosa, Kelly Hearne, Ayoola Adigun, Lisa Farkas, Ryan Cranshaw, Cara Boyarin, Morgan Sawyer, Debora Duro. Influence of Exercise on Body Composition and Patient Outcomes in a Multidisciplinary Pediatric Obesity Clinic. American Journal of Pediatrics. Vol. 6, No. 4, 2020, pp. 488-494. doi: 10.11648/j.ajp.20200604.27

Received: November 10, 2020; Accepted: November 21, 2020; Published: December 16, 2020

\begin{abstract}
Background: Childhood obesity has become a global epidemic, with approximately $34 \%$ of American children affected. This study describes outcomes seen in patients of a Multidisciplinary Pediatric Obesity Clinic (MPOC) who are compliant with individualized exercise regimens conducted by a Clinical Exercise Physiologist (CEP). The goal of this study is to track body composition, physical fitness measures, laboratory results, dietary and behavioral changes over the course of the program. Methods: Prospective cohort study design was implemented. There were 8-week exercise sessions conducted. Participants were evaluated by the CEP before, after the 8-week session using a standardized physical fitness evaluation, measurement of body composition and circumferences. Results: Statistical significance was observed with the increase in height $(p<0.001)$, weight $(p=0.014)$, Total Lean Mass $(p=0.015)$. Increase in percent lean mass for all ethnicities in the study group i.e. black (male and female), hispanic (male and female) and white (male). An increase in the number of push-ups $(\mathrm{p}=0.012)$ was also observed. Conclusion: We can conclude that the use of an MPOC, CEP, and an exercise program early in childhood can potentially prevent the complications of obesity later in life.
\end{abstract}

Keywords: Multidisciplinary, Obesity, Pediatrics, Physical Activity

\section{Introduction}

Childhood obesity is a global epidemic that has more than tripled in the last 40 years, with almost $20 \%$ of children ages 2 18 categorized as obese [1]. According to the most recent National Survey of Children's Health, Florida ranks fourth in the nation for childhood obesity [1]. To combat childhood obesity, our institution has formed the Multidisciplinary Pediatric Obesity Clinic [2, 3] (MPOC), offering patients ages 2-18 an individualized treatment program, developed by an expert team of pediatric gastroenterologists, endocrinologists, dieticians and psychologists. The MPOC team primarily focuses on screening and diagnosing pathological causes of obesity, however, nutritional goal-setting and a psychological assessment to screen for eating patterns, sleep hygiene behaviors, and depressive and suicidal symptoms follows.

Physical activity is an essential component to obesity management. Obese children have been shown to be more respondent to exercise than normal weight peers $[4,5]$. However, it has been shown that physicians do not receive comprehensive exercise and physical activity education during their training [6-10]. In fact, only $13 \%$ of physicians receive physical activity education as it relates to overall health in medical school, with only $6 \%$ of medical schools having a core 
course requirement relating specifically to physical activity [11].

Clinical Exercise Physiologists (CEP) are healthcare professionals with a minimum of a bachelor's degree in exercise science who have specific experience and education in prescribing exercise for lifestyle management. While inclusion of a CEP within pediatric obesity treatment programs is not always common, we believe it is an essential part of treatment to ensure improvement in health outcomes, establishment, and maintenance of a healthier lifestyle. This paper describes the experience of adding a CEP to the MPOC clinical team and outcomes of an 8-week structured exercise program for MPOC patients.

\section{Methods}

\subsection{Study Design}

The project took place at a hospital-based physician practice, the location of the Multidisciplinary Pediatric Obesity Clinic (MPOC). The MPOC consists of an expert team of pediatric gastroenterologists, endocrinologists, dieticians, and psychologists, with the addition of the CEP. The institution is the region's safety-net provider, which works to meet the needs of a 275-square mile area of urban and suburban populations, regardless of ability to pay. The study employed a prospective, cohort design.

The MPOC worked with the hospital's Wellness Center to staff the MPOC with a CEP for the duration of the project. The hospital's Wellness Center is a 10,000 square foot, hospital-based fitness center with professionals certified by the American College of Sports Medicine and the National Strength and Conditioning Association. In addition to the project described, the Wellness Center offers a full range of services including fitness classes, personal training, nutritional counseling, massage therapy, cardiac and pulmonary rehabilitation, diabetes education, health education, specialty classes and events.

\subsection{Initial Evaluation}

Patients from the MPOC were offered the opportunity to participate in this study which is sponsored by the NASPGHAN Foundation Innovations in Clinical Care Grant. After Institutional Review Board (IRB) approval, 32 participants ages 5-to-17 year old (18 males and 14 females) with BMI greater than or equal to 85 th percentile of age, sex, and sex-specific BMI, from the multidisciplinary pediatric obesity clinic (MPOC) agreed to participate in the exercise program. This sample is representative of the population of children from MPOC. Eligible patients were those between ages 5 to 17 and agreed to participate in the exercise program. They attended the MPOC at least twice in one year (initial and follow-up). Participants were evaluated by the CEP during their initial MPOC visit, and during at least one subsequent visit; MPOC patients visited the clinic between two to four times a year. Upon initial evaluation, the CEP measured body composition using bioelectrical impedance (BEI, BIAS Platinum). BIAS Platinum analyzes lean and fat mass, along with height and weight to determine BMI, total fat mass $(\mathrm{kg})$, total lean mass $(\mathrm{kg})$, percent $(\%)$ fat mass, and percent $(\%)$ lean mass. In addition, body circumferences were measured with a standard tape measure. Circumferences of the abdomen, arm, hips, calf, forearm, mid-thigh, and waist were measured, recorded, and compared to national standards for age and sex; waist-to-hip ratio was also calculated from the measured circumferences.

During the initial evaluation, the CEP also completed a thorough fitness evaluation. Cardiorespiratory fitness was measured using the 3-Minute Step Test, a submaximal test most appropriate for use with children; maximal cardiorespiratory fitness was calculated using accepted equations. Muscular strength and endurance was measured using the Push-Up Test. Finally, patients completed a general flexibility assessment using the Sit-and-Reach test. All physical fitness testing adhered to American College of Sports Medicine standards. Results from all assessments were recorded and compared to national standards for age and sex.

After the evaluation, patients and their families were educated on the importance of physical fitness, especially as it relates to the treatment of obesity. The CEP also set both short and long term fitness goals with patients. Using assessment results and patient goals, the CEP prescribed individualized exercise regimen, consisting of a 5-10 minutes warm-up, 20-60 minutes of aerobic, resistance, neuromotor, and/or sports activities, 5-10 minutes cool down of moderate physical activity, and at least 10 minutes of stretching. Increasing ageappropriate physical activity, like sports participation, dance, or gymnastics classes, and playing outside with the family was also emphasized, with a goal of exercising or participating in some type of aerobic activity three to five days per week. All participants were asked to note the duration and frequency of any physical activity they participated in each week, so it may be tracked over the course of MPOC attendance.

\subsection{Intervention}

Individualized exercise programs were managed during four separate 8 -week periods. Patients were allowed to participate in up to all four of the 8 -week periods if they desired. Workouts took place every Saturday for 8 weeks and each lasted about fifty minutes. Participants met at the wellness center and worked with the CEP in a personal-training atmosphere. Participants were split between two groups at the beginning of the session and there was one trainer assigned to each group. The session was started with a 5- minute warm-up, and then the first group focused on circuit based training with rotating exercises while the second group completed an obstacle course. Circuit exercises included: push-ups, sit-ups, step-ups, squats, lunges, ball toss, fast feet, jumping jacks, and jump rope and were times in 30 second intervals (30 seconds on and 30 seconds rest). Obstacle course exercises included a stability disc, bosu ball, cones, ladders, bicep curls with therabands, calf raises, and jump squats. Halfway through the program, at the 20-minute mark, the groups would switch. A 5minute stretch was performed at the end. After each session, the CEP discussed diet and exercise with the group. There was 
one session where several of the participants' parents joined their children in the exercise.

\subsection{Additional Testing: Blood Sampling}

As part of the MPOC, patients underwent standard of care blood sampling and analysis for blood glucose, total cholesterol, LDL, HDL, triglycerides, AST, and ALT. These were obtained at the start and the end of the 8-week program. All data was recorded in the patient medical record.

\subsection{Statistical Analysis}

Numerical variables were presented as Median and Interquartile Range (IQR) and the categorical variables were displayed as frequency and proportion. Changes in numerical variables between pre and post-intervention were analyzed with the Wilcoxon Signed-Rank Descriptive statistics were calculated for all variables, including median and inter quartile range (IQR), to describe dispersion and variability as the data reported is non-parametric. Data samples were matched if they came from repeated observations of the same subject. All analyses were performed using STATA/IC
Version 16.1 for Windows (Stata Corp LLC, College Station, Texas, USA). A two sided $\mathrm{P}$ value less than 0.05 was considered statistically significant.

\section{Results}

\section{Changes in Primary Outcomes: Body Composition}

Descriptive Statistics and Bivariate Results

Thirty-two subjects participated in the study. The median age for males was 18 years $(56.25 \%)$ and females 14 $(43.75 \%)$. The youngest participant in the study was 5 years old and the oldest was 17 years old. Participants age 5-8 were $34 \%, 9-13$ comprised of $40.62 \%$ and $14-17$ were $25 \%$ of study participants, respectively. Represented ethnicities included Black (43.75\%), Hispanic (46.88\%) and White (9.37\%). There was no white female participants enrolled in the study

In this section, we present the body composition data that was recorded and analyzed from the participants in the study. In Table 1, statistically significant changes were seen with $\mathrm{p}$ value $<0.05$ after using the Wilcoxon sign-ranked test due to the nonparametric distribution of the data.

Table 1. Anthropometrics, Demographics and Cardiorespiratory Fitness before and after Participation in the Multidisciplinary Pediatric Obesity Clinic.

\begin{tabular}{|c|c|c|c|}
\hline \multirow{2}{*}{ Variables } & \multirow{2}{*}{$\begin{array}{l}\text { Pre Intervention } \\
\text { Median (IQR) or Frequency (\%) }\end{array}$} & \multirow{2}{*}{$\begin{array}{l}\text { Post Intervention } \\
\text { Median (IQR) or Frequency (\%) }\end{array}$} & \multirow{2}{*}{ P value* } \\
\hline & & & \\
\hline \multicolumn{4}{|l|}{ Demographics Table } \\
\hline \multicolumn{4}{|l|}{ Gender } \\
\hline Male & $18(56.25)$ & $18(56.25)$ & \\
\hline Female & $14(43.75)$ & $14(43.75)$ & \\
\hline \multicolumn{4}{|l|}{ Age } \\
\hline $5-8$ & $11(34.38)$ & $11(34.38)$ & \\
\hline $14-17$ & $8(25.00)$ & $8(25.00)$ & \\
\hline \multicolumn{4}{|l|}{ Ethnicity } \\
\hline Black & $14(43.75)$ & $14(43.75)$ & \\
\hline Hispanic & $15(46.88)$ & $15(46.88)$ & \\
\hline White & $3(9.37)$ & $3(9.37)$ & \\
\hline \multicolumn{4}{|l|}{ Anthropometrics } \\
\hline \multicolumn{4}{|l|}{ Body Composition } \\
\hline $\operatorname{BMI}\left(\mathrm{kg} / \mathrm{m}^{2}\right)$ & $31.1(5.9)$ & $31.8(10.8)$ & 0.709 \\
\hline Total Fat Mass (kg) & $23.85(13.5)$ & $24.5(6.8)$ & 0.548 \\
\hline Percent (\%) Fat Mass & $36.4(9.2)$ & $33.8(9.8)$ & 0.207 \\
\hline Total Lean Mass (kg) & $43.1(16.5)$ & $44.4(14.7)$ & 0.015 \\
\hline Percent (\%) Lean Mass & $63.2(9.2)$ & $66.2(9.8)$ & 0.221 \\
\hline \multicolumn{4}{|l|}{ Body Circumference } \\
\hline Hips $(\mathrm{cm})$ & $100(16.2)$ & $102(17)$ & 0.693 \\
\hline Waist $(\mathrm{cm})$ & $93.5(11)$ & $94(10)$ & 0.754 \\
\hline Waist-to-Hip Ratio & $0.92(0.13)$ & $0.98(0.11)$ & 0.564 \\
\hline \multicolumn{4}{|l|}{ Cardiorespiratory Fitness } \\
\hline 3 min step test (HR) & $119(32)$ & $117(19)$ & 0.667 \\
\hline Push Up Test (times) & $32(15)$ & $36.5(16)$ & 0.012 \\
\hline Sit and Reach (times) & $22(9)$ & $23.75(14)$ & 0.660 \\
\hline Sit Ups (times) & $35(14)$ & $33.5(11)$ & 0.833 \\
\hline
\end{tabular}

* P value $<0.05$ statistical significance. Wilcoxon signed-rank test done for nonparametric distribution.

Anthropometrics: Some anthropometric changes reached statistical significance when comparing before and after the intervention. Height $(\mathrm{cm})$, weight $(\mathrm{kg})$, BMI $\left(\mathrm{kg} / \mathrm{m}^{2}\right)$, Total Fat Mass (kg), Total Lean Mass (kg) and Percent Lean Mass, Hips, Waist circumference and Waist-to-Hip ratio increased while Percent Fat Mass decreased. Statistical significance was observed with the increase in height $(p<0.001)$, weight $(\mathrm{p}=0.014)$ and Total Lean Mass $(\mathrm{p}=0.015)$.

It is important to note however, that overall, patients showed a decrease in total fat mass for females (black and hispanic) a 
decrease in percent fat mass for males (black and hispanic) and females (black and hispanic) with no change in white (males). There was an increase in total lean mass for males (black and hispanic) and female (black) and no change in total lean mass for hispanic females. There was increase in percent lean mass for all ethnicities in the study group i.e. black (male and female), hispanic (male and female) and white (male). (Figure 1).

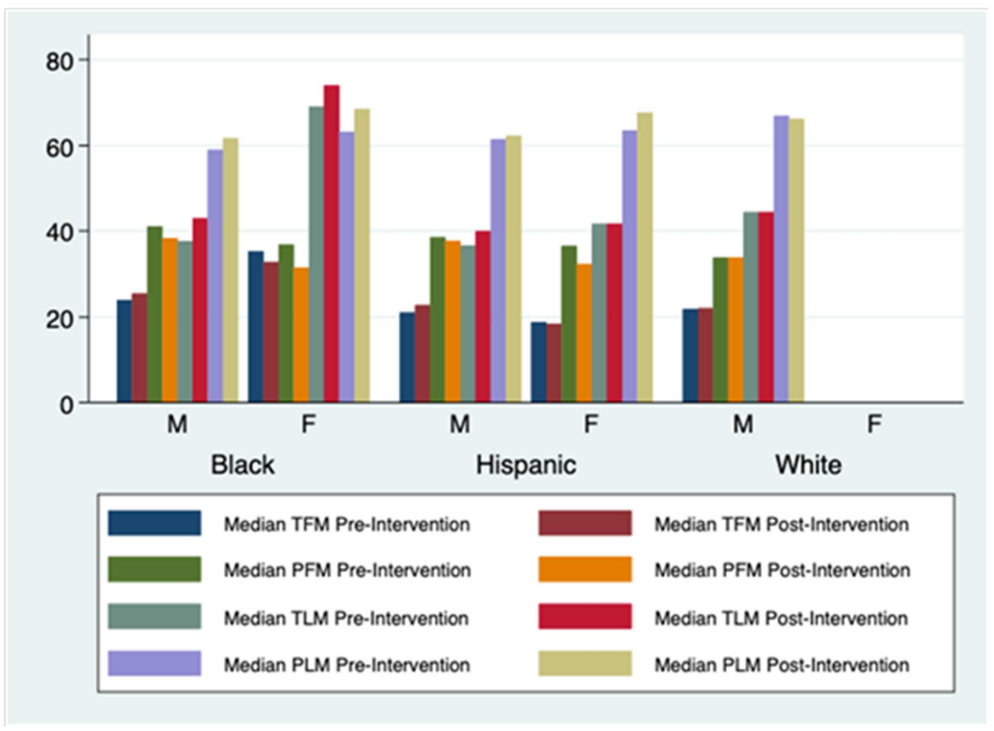

Figure 1. Average changes in body composition before and after 8-week exercise program represented as a bar graph.

*TFM - Total Fat Mass, PFM - Percent Fat Mass, TLM - Total Lean Mass, PLM - Percent Lean Mass.

M- Male, F- Female.

Figure 2 shows the changes in laboratory values for males and females grouped by ethnicity. There was an increase in median glucose values for males and females (black) a decrease in glucose males and females (hispanic), decrease in total cholesterol for males (hispanic) and a decrease in Triglycerides for females (black and hispanic)

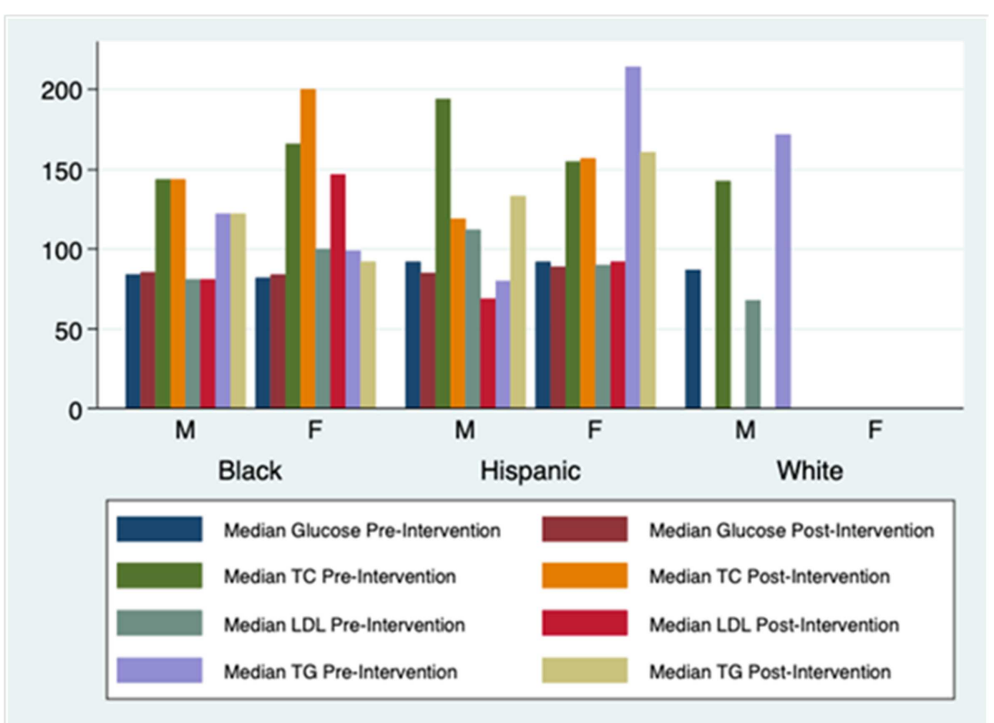

Figure 2. Average Changes in Laboratory Values for Participants Before and After Intervention.

*TC - Total Cholesterol, LDL - Low Density Lipoprotein, TG- Triglyceride. $\mathrm{M}$ - Male, F- Female.

\section{Changes in Secondary Outcomes: Cardiorespiratory} Fitness, Muscular Strength, and Nutrition

Cardiorespiratory Fitness: A reduction in the 3 minutes step test and number of sit-ups after the completion of the 8 week exercise program was observed. And an increase in the number of push-ups and sit-and-reach was also observed. (Table 1)

Aside from body compositions, body circumference measurements were recorded, and these did not change significantly before and after the exercise program for study participants. In terms of cardiorespiratory fitness, we looked 
closely at the participants' ability to increase the number of actual exercises they were able to complete at the preexercise program session compared to the post-exercise evaluation. Overall, patients were able to increase the number of push-ups they were able to perform $(\mathrm{p}=0.012)$ (Table 1).

Table 2. Laboratory Results of Patients Before and After Participation in Intervention Program.

\begin{tabular}{llll}
\hline \multirow{2}{*}{ Variables (mg/dl) } & Pre Intervention & Post Intervention & P value \\
\cline { 2 - 3 } & Median (IQR) & Median (IQR) & \\
\hline Total Cholesterol & $151(51)$ & $153(23)$ & 0.094 \\
Glucose & $85.5(10.5)$ & $88(8)$ & 0.118 \\
HDL & $45(17)$ & $41(6)$ & 0.631 \\
LDL & $86(44)$ & $86(11)$ & 0.778 \\
TG & $122(80)$ & $133(19)$ & 0.015 \\
\hline
\end{tabular}

* HDL=High Density Lipoprotein, LDL=Low Density Lipoprotein, $\mathrm{TG}=$ Triglyceride.

* P value $<0.05$ statistical significance. Wilcoxon signed-rank test done for nonparametric distribution.

Although not the primary purpose of this research, we documented before and after exercise program laboratory results which were ordered by the pediatric gastroenterologist. Specific studies included glucose level, total cholesterol, HDL cholesterol, LDL cholesterol, and triglycerides. In Table 2, we see an increase in the median for all the laboratory values except LDL which remained the same.

Exercise Program, Duration and Frequency: All participants in the study enrolled in the exercise activities according to their age and thus were divided into two camps namely kids (0-12 years) and teens ( $\geq 13$ years) camp activities. The duration of the exercise programs lasted for 4 quarters; Quarter 1 - January to March 2019, Quarter 2 - April to June 2019, Quarter 3 - June to August 2019, Quarter 4 - September to November 2019. Kids were the only participants for the $1^{\text {st }}$ quarter and had a testing day on January before starting the program to evaluate their resting heart rate and oxygen saturation. They had 8 exercise sessions and on the final sessions, the resting heart rate and oxygen saturation were measured. During the $2^{\text {nd }}$ quarter, teens ( $\geq 13$ years) were included in the study along with their own exercise sessions along with the kids. Also, a total of 8 sessions were completed with evaluations done at the first and last sessions. The 2 group of participants i.e. kids and teens participated in the exercise programs with kids enrolling in quarters $1-4$ and teens in quarters $2-4$. Table 3 describes the exercise in detail for kids and teens divided into quarters, dates, and exercise activities.

Table 3. Description of the Various Types of Exercise Programs Done at the Multidisciplinary Pediatric Obesity Clinic.

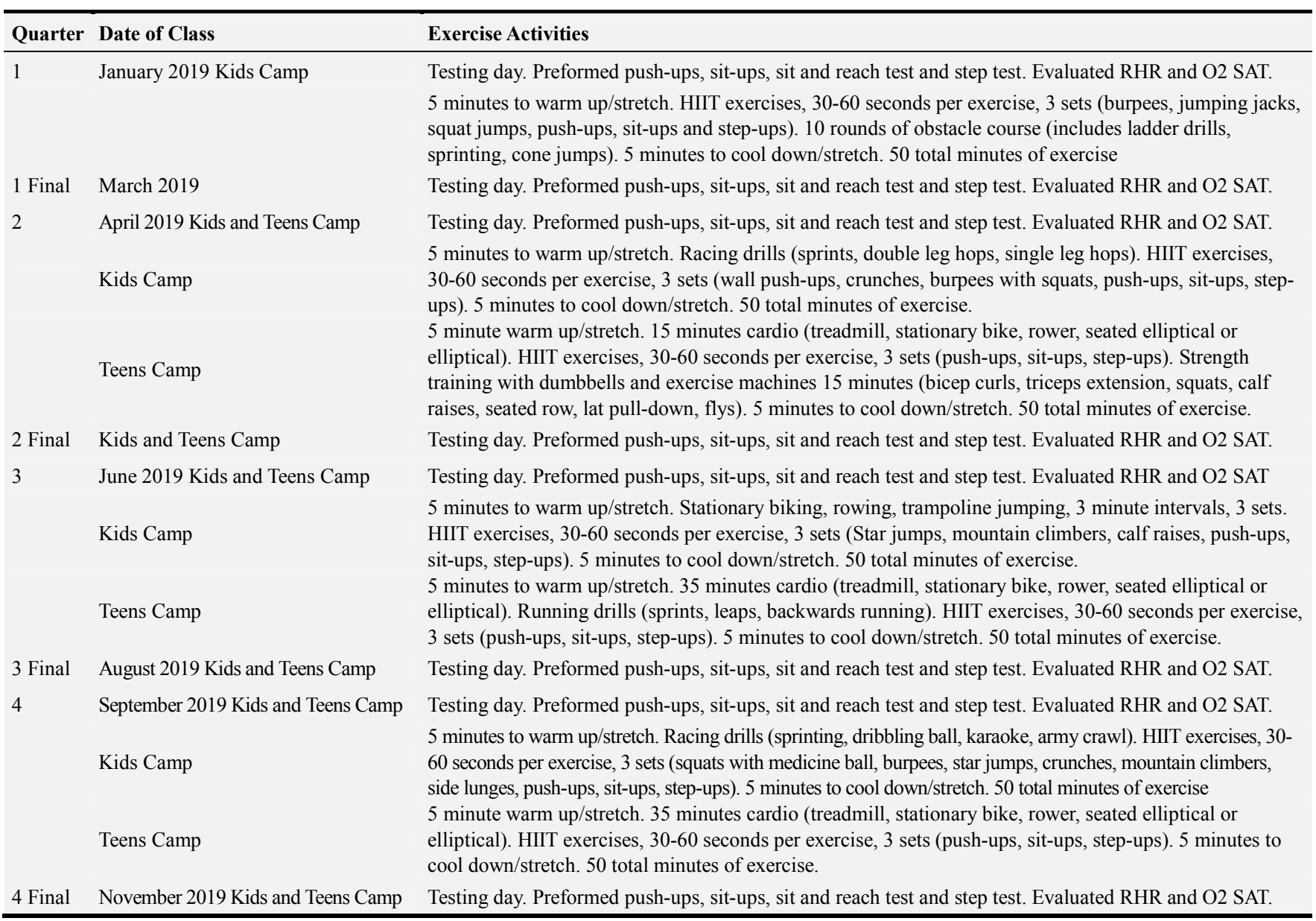

HIIT- High Intensity Interval Training, RHR - Resting Heart Rate, O2 SAT- Oxygen Saturation. 


\section{Discussion}

In the present study, the overall intervention outcomes suggest that the exercise program was effective in improving anthropometric and cardiorespiratory fitness. Increases in total and percent lean mass suggest a positive effect of the intervention on body composition. Different ethnic groups have varying degrees of fatty liver and metabolic syndrome. It is well known that individuals of Hispanic and Asian ethnicity have more levels of fatty liver compared to African Americans [12, 13]. In the study, percent lean mass decreased in both African Americans and Hispanics further suggesting the benefits of patients participating in MPOC. There might have been high levels of insulin and metabolic barriers that prevented males (black, hispanic and white) from having a reduction in total and percent fat mass.

The nutrition of some participants changed with the exercise program. Participants increased their vegetable and fruit intake, incorporating salads, additional vegetables at meals and also making better snack choices. Some participants started bringing their own lunches to school as well as eating breakfast. In general, participants have reduced intake of sugar-sweetened beverages. This shows that the use of a holistic multidisciplinary team, including a CEP, can have a significant impact on a child's health and well-being. Parents across the board were interviewed and noted that behavioral changes were also being made at home.

Some patients had a combination of comorbidities. One patient had prediabetes, Acanthosis Nigricans, Premature Adrenache, and Attention Deficit Hyperactivity Disorder. Another patient had liver steatosis non-alcoholic liver disease, and metabolic syndrome. There was a history of obstructive hydrocephalus and craniopharyngioma in another patient. Lastly, a diagnosis of Non-Alcoholic Fatty Liver Disease (NAFLD) and Vitamin D Deficiency was present in a patient.

The waist circumference in patients which is superior to BMI and previous literature has shown that BMI fails to determine abdominal adiposity $[14,15]$ remained the same before and after the exercise program.

Secondary outcomes were also measured during the study examining cardiorespiratory fitness, muscular strength and nutrition. Physical activities like push-ups, 3 minutes test and sit-and-reach showed improvement in a majority of our participants when measured before and after the exercise intervention program. Cardiorespiratory fitness measured using the three minute step test [16] showed a decrease in resting heart rate. This was performed by the CEP who was responsible for tracking changes in physical fitness, exercise duration and frequency during the 8-week program. There were select participants who needed additional coaching and/or behavioral intervention to encourage them to exercise. The exercise physiologist and program team tried to make the program as fun and rewarding as possible, ensuring all exercise prescribed were age-appropriate and could be completed at home, to maintain results long-term.
Additionally, program staff recommended extracurricular activities to program participants; after recommendations, many participants joined recreational sports like soccer and basketball.

The current study has a number of limitations. First, although we attempted to recruit more than enough participants with BMI greater than or equal to 85 th percentile we recognize the potential bias in a small sample size of 32 participants and future studies may wish to compensate for this bias by recruiting larger samples. With the increasing diversity of the United States [17], our findings can be generalizable depending on the demographics or diversity of the geographic location.

\section{Conclusion}

In conclusion, the prevalence and incidence of childhood obesity as an epidemic [18] in the United States can be mitigated or reduced with the use of a multidisciplinary pediatric obesity clinic. The incorporation of exercise programs early in childhood can change the daily lifestyle and habit of children so they can live a healthier life void of the chronic metabolic diseases that come with childhood obesity. Our prospective study can serve as a template or pilot study for other obesity clinics across the nation to actively engage their patients in exercise intervention programs similar to ours. Future research should consist of prospective studies with close follow-up of participants to ensure compliance with change in lifestyle from sedentary to more active and engaging physical activities.

\section{Funding Source}

NASPGHAN Foundation, Clinical Innovation Grant.

\section{References}

[1] Hales CM, Carroll MD, Fryar CD, Ogden CL. Prevalence of Obesity Among Adults and Youth: United States, 2015-2016. NCHS Data Brief. 2017; (288): 1-8.

[2] Novick MB, Wilson CT, Walker-Harding LR. Potential solutions for pediatric weight loss programs in the treatment of obesity in rural communities. Transl Behav Med. 2019; 9 (3): 460-467. doi: 10.1093/tbm/ibz030.

[3] Bischoff SC, Boirie Y, Cederholm T, et al. Towards a multidisciplinary approach to understand and manage obesity and related diseases. Clin Nutr. 2017; 36 (4): 917-938. doi: 10.1016/j.clnu.2016.11.007.

[4] Mitchell, J. A., Pate, R. R., Beets, M. W., \& Nader, P. R. (2013). Time spent in sedentary behavior and changes in childhood BMI: a longitudinal study from ages 9 to 15 years. International journal of obesity, 37 (1), 54-60.

[5] Mitchell, J. A., Rodriguez, D., Schmitz, K. H., \& AudrainMcGovern, J. (2013). Greater screen time is associated with adolescent obesity: a longitudinal study of the BMI distribution from ages 14 to 18 . Obesity, 21 (3), 572-575. 
[6] Smith AW, Borowski LA, Liu B, et al. U.S. primary care physicians' diet-, physical activity-, and weight-related care of adult patients. Am J Prev Med. 2011; 41 (1): 33-42. doi: 10.1016/j.amepre.2011.03.017.

[7] Van Wormer J, Pronk N, Kroeninger G. Clinical counseling for physical activity: translation of a systematic review into care recommendations. Diabetes Spectrum. 2009; 22: 48-55.

[8] Frank E, Segura C. Health practices of Canadian physicians. Can Fam Physician. 2009; 55: 810-811.e7.

[9] Epel OB, Ziva Regev M. Quality and correlates of physical activity counseling by health care providers in Israel. Prev Med. 2000; 31: 618-626.

[10] Van der Ploeg HP, Smith BJ, Stubbs T, Vita P, Holford R, Bauman AE. Physical activity promotion-are GPs getting the message? Aust Fam Physician. 2007; 36: 871-874.

[11] Dacey, M. L., Kennedy, M. A., Polak, R., \& Phillips, E. M. (2014). Physical activity counseling in medical school education: a systematic review. Medical education online, 19 (1), 24325.

[12] Szanto KB, Li J, Cordero P, Oben JA. Ethnic differences and heterogeneity in genetic and metabolic makeup contributing to nonalcoholic fatty liver disease. Diabetes Metab Syndr Obes. 2019; 12: 357-367. Published 2019 Mar 19. doi: 10.2147/DMSO.S182331.
[13] Pan, J. J., \& Fallon, M. B. (2014). Gender and racial differences in nonalcoholic fatty liver disease. World journal of hepatology, 6 (5), 274.

[14] Javed, A., Jumean, M., Murad, M. H., Okorodudu, D., Kumar, S., Somers, V. K.,... \& Lopez-Jimenez, F. (2015). Diagnostic performance of body mass index to identify obesity as defined by body adiposity in children and adolescents: a systematic review and meta-analysis. Pediatric obesity, 10 (3), 234-244.

[15] Barreira, T. V., Broyles, S. T., Gupta, A. K., \& Katzmarzyk, P. T. (2014). Relationship of anthropometric indices to abdominal and total body fat in youth: sex and race differences. Obesity, 22 (5), 1345-1350.

[16] Jankowski M, Niedzielska A, Brzezinski M, Drabik J. Cardiorespiratory fitness in children: a simple screening test for population studies. Pediatr Cardiol. 2015; 36 (1): 27-32. doi: 10.1007/s00246-014-0960-0.

[17] Colby, S. L., \& Ortman, J. M. (2017). Projections of the size and composition of the US population: 2014 to 2060: Population estimates and projections.

[18] Kumar S, Kelly AS. Review of Childhood Obesity: From Epidemiology, Etiology, and Comorbidities to Clinical Assessment and Treatment. Mayo Clin Proc. 2017; 92 (2): 251-265. doi: 10.1016/j.mayocp.2016.09.017. 